\section{A Reinvestigation of Maximal Signal Drop in Dynamic Susceptibility Contrast Magnetic Resonance Imaging}

Yi-Jui Liu, MS

Hsiao-Wen Chung, PhD

Ing-Jye Huang, BS

Fu-Nien Wang, MS

Shy-Chyi Chin, MD

Chang-Shin Lee, PhD

Cheng-Yu Chen, MD

\section{A B S T R A C T}

Background and Purpose. The purpose of this study was to reevaluate the usefulness of relative maximum signal drop (rMSD), as compared to relative cerebral blood volume (rCBV) and cerebral blood flow (rCBF), in dynamic susceptibility contrast magnetic resonance imaging (MRI). Methods. Twenty-five patients (11 with cerebral gliomas and 14 with infarcts of middle cerebral arterial territories) were included. The rMSD values were measured from 83 regions of interest and compared with measurements from corresponding rCBV and rCBF maps. Results. In stroke patients, rMSD correlated strongly with rCBF $(r=0.96)$ but only fairly with $\mathrm{rCBV}(r=0.69)$. The absence of an association between $\mathrm{rMSD}$ and $\mathrm{rCBV}$ was evident in regions of increased contrast bolus dispersion. In glioma patients, the correlation of $r M S D$ with $r C B F(r=0.85)$ was similar to that of $r M S D$ with rCBV $(r=0.80)$. The interparameter associations were well predicted by computer simulations. Conclusions. The authors conclude that rMSD is as useful as rCBF under a variety of pathophysiological conditions, whereas in conditions with normal mean transit time, such as brain tumors, rMSD provides equivalent blood volume information to rCBV. The simplicity of rMSD maps could lead to the increased use of perfusionweighted MRI.

Key words: Maximal signal drop, cerebral blood volume, cerebral blood flow, dynamic susceptibility contrast magnetic resonance imaging.

Liu Y-J, Chung H-W, Huang I-J, Wang F-N, Chin S-C, Lee C-S, Chen C-Y. A reinvestigation of maximal signal drop in dynamic susceptibility contrast magnetic resonance imaging. J Neuroimaging 2002;12:330-338.

DOI: $10.1177 / 105122802237976$

Dynamic perfusion-weighted magnetic resonance imaging (MRI) using the intravenous injection of paramagnetic contrast agents is now considered an important tech- nique to assess cerebral hemodynamics in various brain diseases. ${ }^{1,2}$ This technique allows the use of several relative perfusion parameters to represent different tracer kinetic aspects of microcirculation status, such as relative cerebral blood volume (rCBV), relative cerebral blood flow ( $\mathrm{rCBF})$, and relative mean transit time (rMTT), during the first-pass tracer transit. ${ }^{3,4}$ In cerebral gliomas, for instance, a focal increase in $\mathrm{rCBV}$ correlates with an increase in tumor angiogenesis, which is an important pathological indicator for tumor activity and grading. ${ }^{5,6}$ Maps of rCBV generated from dynamic perfusionweighted magnetic resonance images are therefore helpful in guiding stereotactic biopsy sites for brain tumors. ${ }^{7}$ One minor disadvantage of the rCBV method, however, lies in the need for customized programming on numerical integration, which somewhat curtails its wide use by radiologists.

A simple method using relative maximum signal drop (rMSD) in a dynamic perfusion-weighted MRI series to represent $\mathrm{rCBV}$ has recently been proposed to remedy the disadvantage mentioned above. ${ }^{8}$ In this study, rMSD was shown to highly correlate with $\mathrm{rCBV}$ in patients with various intracranial mass lesions, justifying the eligibility of this new parameter. In addition, the method to calculate $\mathrm{rMSD}$ can easily be integrated into general clinical

Received February 11, 2002, and in revised form May 28, 2002. Accepted for publication June 3, 2002.

From the Department of Electrical Engineering, National Taiwan University, Taipei (YJL, HWC, IJH, FNW); and the Department of Radiology, Tri-Service General Hospital and National Defense Medical Center, Taipei, Taiwan (HWC, SCC, CSL, CYC).

Address correspondence to Cheng-Yu Chen, MD, Department of Radiology, Tri-Service General Hospital and National Defense Medical Center, 325, Sec 2, ChengKung Road, Neihu 114, Taipei, Taiwan, ROC. E-mail: sandy0928@seed.net.tw. 
magnetic resonance systems without the need for complicated programming. Similar results were also reported in an earlier study on patients with cerebral gliomas. ${ }^{9}$ It was further demonstrated that rMSD exhibited better noise immunity compared with rCBV obtained via the integration method. ${ }^{8}$ For radiologists, therefore, rMSD seems to be a convenient alternative to numerical integration in the assessment of rCBV.

The current study further investigates rMSD in dynamic perfusion-weighted MRI in an attempt to provide a basis for a better understanding of its utility. A simplified theoretical analysis on tracer kinetics was first attempted, followed by a retrospective clinical study and computer simulations for a confirmation. We sought to test the utility of rMSD in diseases with different hemodynamics, such as cerebral gliomas and ischemic cerebral stroke, by comparing the correlations between rMSD and $\mathrm{rCBV}$ or $\mathrm{rCBF}$ in dynamic susceptibility contrast perfusion MRI.

\section{Materials and Methods}

\section{Background}

With contrast injection, information on brain perfusion can be obtained by evaluating the dynamic changes of magnetic resonance signal intensity caused by the susceptibility effect. Specifically, T2* is reduced, and the signal intensity drops in $\mathrm{T} 2{ }^{*}$-weighted images. MSD is the difference between the precontrast and the lowest signal intensities during the first passage of contrast bolus (Fig 1A). Previous studies have shown that the change in apparent transverse relaxation rate $\left(\Delta\left[1 / \mathrm{T} 2^{*}\right]=\Delta \mathrm{R} 2^{*}\right)$ is proportional to contrast concentration in the brain tissue, ${ }^{3}$ which in turn can be expressed as a function of the magnetic resonance signal:

$$
\Delta \mathrm{R} 2 *(t) \propto C(t)=-\frac{k}{T E} \ln \left(\frac{S(t)}{S_{0}}\right),
$$

where $k$ is a constant (depending on tissue types, pulse sequences, and field strength), $S(t)$ is signal intensity at time $t, S_{0}$ is precontrast signal intensity, and $C(t)$ is the first-pass tissue tracer concentration as a function of time. Equation 1 can be used to convert the signal-time curve into the relative concentration-time curve, in which the first-pass transit is generally modeled as a gamma variate function (Fig 1B)..$^{10,11}$ In case the contrast agent remains intravascular, $\mathrm{rCBV}$ at a specific location is proportional to the total amount of tracer flowing through that region; that is, it is proportional to the integrated area under $C(t)^{3}$ :

$$
\mathrm{rCBV}=\int C(t) d t
$$

In the simplest approximation, the gamma variate curve resembles a triangular shape, as shown in Figure 1C. Therefore, $\mathrm{rCBV}$, the integrated area, is proportional to the curve width of $C(t)$ multiplied by the maximum concentration, $C_{\max }$ :

$$
\mathrm{rCBV} \propto \text { width } \cdot C_{\text {max }}
$$

The maximum concentration in turn is related to $\mathrm{rMSD}$ in dynamic perfusion-weighted MRI by the following relationship from equation 1 :

$$
C_{\max }=-\frac{k}{T E} \ln \left(\frac{S_{\min }}{S_{0}}\right)=-\frac{k}{T E} \ln \left(1-\frac{\mathrm{MSD}}{S_{0}}\right)
$$

where $S_{\min }$ is the lowest signal intensity during the firstpass bolus transit. In other words, in a single perfusionweighted MRI examination for which $k, T E$, and $S_{0}$ are fixed, rMSD seems to be closely related to the maximum concentration, $C_{\max }$, rather than $\mathrm{rCBV}$, which is equal to the product of $C_{\max }$ and the curve width of $C(t)$ (see equation 2). It is therefore anticipated that the rMSD method proposed to represent $\mathrm{rCBV}^{8,9}$ should correlate well with rCBV only under the assumption that the contrast agent bolus dispersion, that is, the width of the first-pass tracer concentration-time curve, remains largely unaltered. This prerequisite includes a well-controlled contrast injection, such as the use of a power injector to eliminate variations in the perfusion-curve dispersion due to differences in injection speed (ie, nonideal bolus), plus that the disease of interest shows the least possible change in contrast bolus dispersion. In cases in which prolonged rMTT is present, leading to a widened $C(t)$ curve, rMSD may fail to reliably indicate $\mathrm{rCBV}$ information.

On the other hand, we consider that rMSD should correlate better with $\mathrm{rCBF}$ than with $\mathrm{rCBV}$. The $\mathrm{rCBF}$ is related to $\mathrm{rCBV}$ through division by $\mathrm{rMTT}$ :

$$
\mathrm{rCBF}=\frac{\mathrm{rCBV}}{\mathrm{rMTT}},
$$

where rMTT is defined as the normalized first moment of $C(t)$ :

$$
\mathrm{rMTT}=\frac{\int t \cdot C(t) d t}{\int C(t) d t}
$$

In other words, rMTT represents the time interval from bolus arrival $(t=0)$ to the centroid of the $C(t)$ curve. For typical $C(t)$ curves showing fast wash-in and slow washout, this time interval often parallels the width of $C(t){ }^{12}$ Therefore, $C_{\max }$ (ie, the height of $C[t]$ ), equaling the area divided by the width, would be directly related to $\mathrm{rCBF}$. 


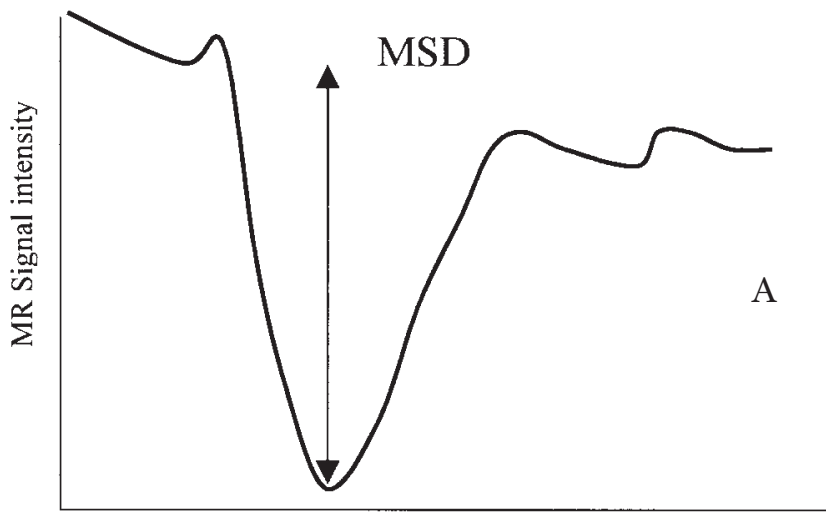

Time after injection

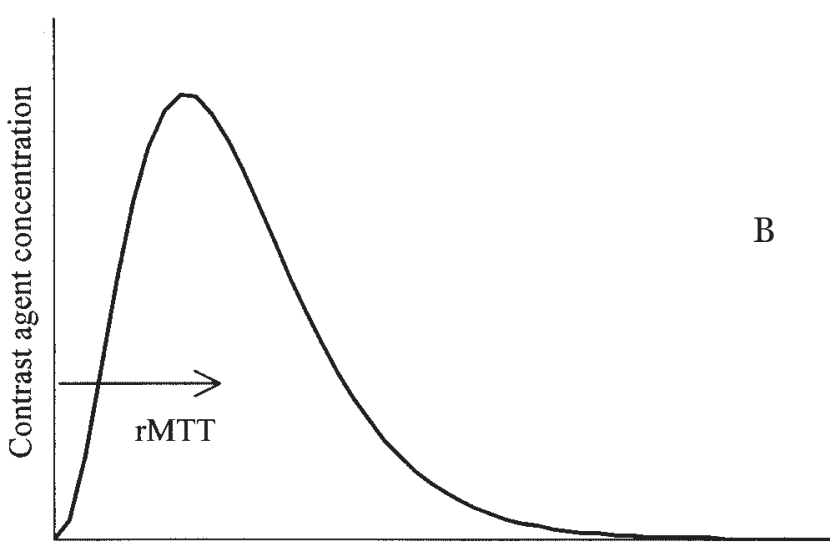

Time after injection

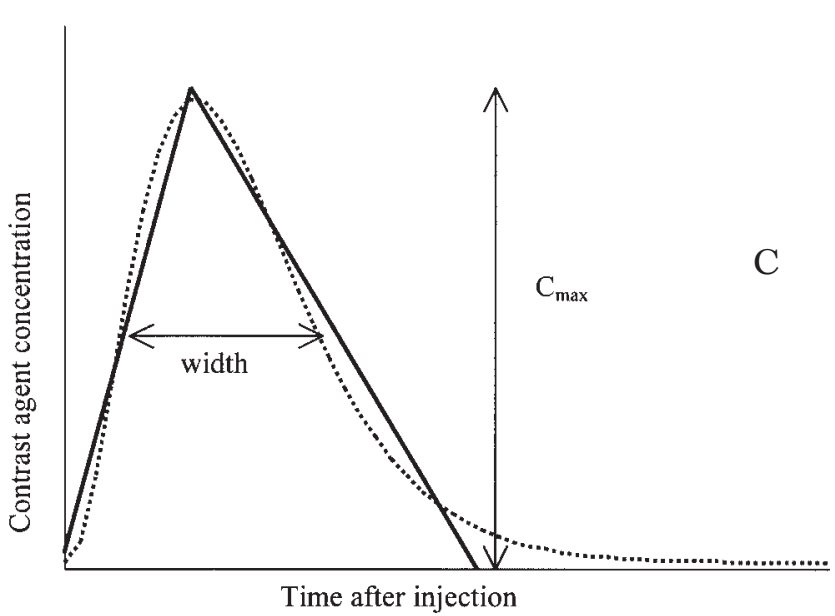

Fig 1. Signal-time curve and concentration-time curves following injection of an intravascular tracer. (A) Graph shows the time course of magnetic resonance (MR) signal intensity during passage of contrast bolus. Maximum signal drop (MSD) is determined by the difference between precontrast and the lowest signal intensity during passage of contrast bolus. (B) Graph shows the shape of a typical first-pass transit, which can generally be modeled as a gamma variate function. rMTT = relative mean transit time. (C) Graph shows that the first-pass concentration-time curve in A resembles a triangular shape in a simplified approximation. The relative cerebral blood volume, defined as the integrated area under the curve, is therefore roughly proportional to the curve width multiplied by the maximum concentration.
As a consequence, rMSD should correlate well with $\mathrm{rCBF}$, regardless of possible changes in rMTT. Also note that all perfusion parameters referred to above are of relative values only rather than absolute quantifications. ${ }^{13}$

\section{Computer Simulations}

To consolidate our inference, we performed computer simulations to examine the theoretical correlation between rMSD and $\mathrm{rCBV}$, as well as that between rMSD and $\mathrm{rCBF}$. For this purpose, concentration-time curves were mathematically created by convolution of an arterial input function with tissue residue functions, ${ }^{12}$ following which a series of signal-time curves were computed using equation 1. A gamma variate function was used for the arterial input function, ${ }^{14}$ whereas the tissue residue function was modeled as an exponential decaying function scaled by CBF with time-constant MTT to represent a single well-mixed vascular compartment. ${ }^{15}$ A total of 1000 combinations of $\mathrm{CBF}$ and MTT were used to generate tissue signal-time curves covering a wide range (more than an order of magnitude) of pathophysiological hemodynamic situations. Interframe noise ${ }^{16}$ was subsequently added. The perfusion parameters were then calculated and normalized by that of the simulated normal tissue. Finally, the relationships between perfusion parameters were graphically examined.

\section{Patients and Image Acquisition}

MRI data from 25 patients, 11 with cerebral gliomas $(9$ men and 2 women aged 24-75 [48.8 \pm 14.7$]$ years) and 14 with acute infarcts of middle cerebral arterial territories $(8$ men and 6 women aged 22-81 [54.9 \pm 18.2$]$ years), were retrospectively investigated. All patients underwent perfusion examinations via dynamic susceptibility contrast MRI on a 1.5-T system (Siemens Magnetom Vision+, Erlangen, Germany). A series of perfusion-weighted images was acquired using the echo-planar imaging technique $(\mathrm{TE}=44 \mathrm{~ms}, \mathrm{FOV}=230 \mathrm{~mm}$, matrix size $=102 \times$ 128 , slice thickness $=5 \mathrm{~mm}, 6$ slices in each measurement), following intravenous injection of $0.2 \mathrm{mmol} / \mathrm{kg}$ gadopentetate (Magnevist, Schering, Germany) at a speed of about 4 to $5 \mathrm{~mL} / \mathrm{sec}$ through a 20-gauge angiocatheter inserted in the antecubital vein. Seventy-five frames were obtained at 1-second intervals for each slice to encompass the first-pass tracer transit. Images were digitally transferred to a personal computer for pixel-by-pixel analysis of perfusion parameters.

\section{Data Analysis}

The MSD values were obtained as described previously, ${ }^{8}$ the procedure for which will not be detailed here. Equations 1 to 6 were used to compute rCBV, rMTT, and 

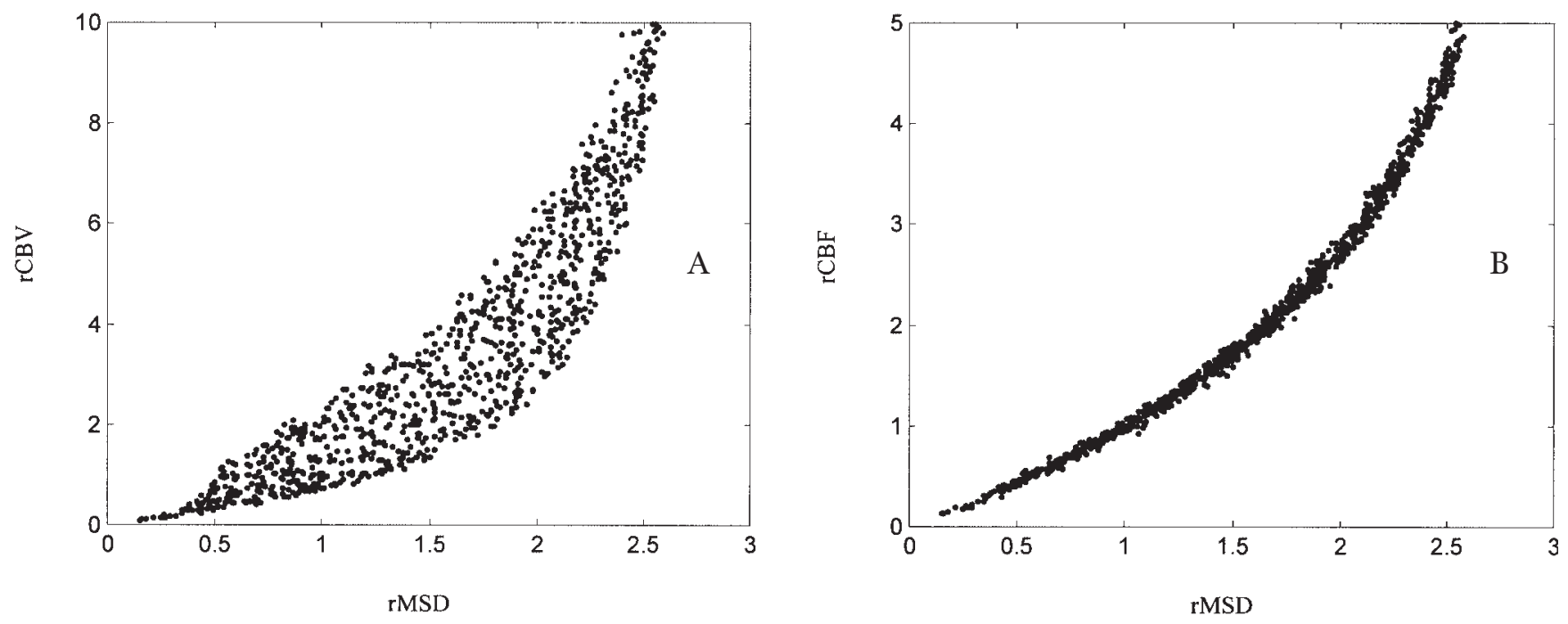

Fig 2. Relationships obtained from computer simulations between relative maximum signal drop and 2 cerebral perfusion parameters. (A) Plot shows relative maximum signal drop (rMSD) versus relative cerebral blood volume ( $r C B V)$. Although these 2 parameters were positively correlated, scattering in the data indicated that a prediction of rCBV using rMSD could lead to substantial inaccuracies. (B) Plot shows rMSD versus relative cerebral blood flow (rCBF). A strong correlation was found regardless of the wide spread in concentration-time curve morphology, suggesting that rMSD reliably indicates rCBF information.

rCBF. Second-pass effects were minimized via an examination of the intensity change after the concentration peak. If the signal intensity rose above a certain threshold (90\% of the precontrast baseline intensity), all data after this time point were excluded. Thus, 4 parameter maps (including $\mathrm{rMSD}$ ) were generated for each slice location.

Regions of interest (ROIs) were manually encircled to analyze the rMSD correlation with $\mathrm{rCBV}$ and $\mathrm{rCBF}$. This included 2 to 5 ROIs selected from each patient, depending on the spatial extent, topographic distribution, and lesion nature. Normalization with respect to normal brain parenchyma was performed for all parameters to minimize inevitable interpatient variations due to factors such as system gain or cardiac output. ROIs showing abnormal signal-time response, that is, with a single broad hump obviously covering more than 1 transit, were not included. Consequently, 83 ROIs (39 from glioma patients and 44 from stroke patients) were obtained. The relationships between $\mathrm{rMSD}$ and $\mathrm{rCBV}$ as well as between $\mathrm{rMSD}$ and $\mathrm{rCBF}$ were then examined using Pearson's correlation coefficient. The difference in rMTT between the two groups of patients was assessed using Student's $t$ test.

\section{Results}

Figures 2A and 2B show the rMSD-rCBV and rMSDrCBF relationships, respectively, obtained from the computer simulations. One notices in Figure 2A that although
rMSD is associated with $\mathrm{rCBV}$, as reported previously, ${ }^{8,9}$ there exists significant scattering in the data, indicating that the prediction of $\mathrm{rCBV}$ using $\mathrm{rMSD}$ could result in large errors. On the other hand, rMSD and $\mathrm{rCBF}$ show a very close association, even with the wide range of $\mathrm{CBF}$ and MTT values chosen in the simulation procedure (Fig $2 \mathrm{~B}$ ), confirming our theoretical analysis stated previously.

Figure 3A shows rMSD plotted versus rCBV for the data from patients with intracranial tumors (filled circles) and from patients with ischemic stroke (open squares). The good correlation found for the tumor group (Pearson's correlation coefficient $r=0.80, \mathrm{n}=39$ ) is in agreement with that reported by $\mathrm{Cha}$ et $\mathrm{al},{ }^{8}$ although the regression slope appears higher in our study, likely because of our use of a higher contrast dose, resulting in longer injection time. For stroke patients, however, the correlation between rMSD and $\mathrm{rCBV}$ was found to be only fair (Fig 3A, open squares; $r=0.69, \mathrm{n}=44$ ). The representative value of $\mathrm{rMSD}$ for $\mathrm{rCBV}$ therefore seems to be lower on patients with cerebral ischemia compared with intracranial tumors. Figure 3B shows the rMSDrCBF relationships, the associations of which were found to be high for both groups of patients $(r=0.85$ and 0.96 for tumor and stroke patients, respectively). One in particular notices for stroke patients (Figs 3A and 3B, open squares) the excellent prediction value of $\mathrm{rMSD}$ for $\mathrm{rCBF}$ versus the large scattering in the rMSD-rCBV correlation. The experimental interparameter correlations are similar in numerical value to the simulation data in Figures $2 \mathrm{~A}$ and $2 \mathrm{~B}$. 

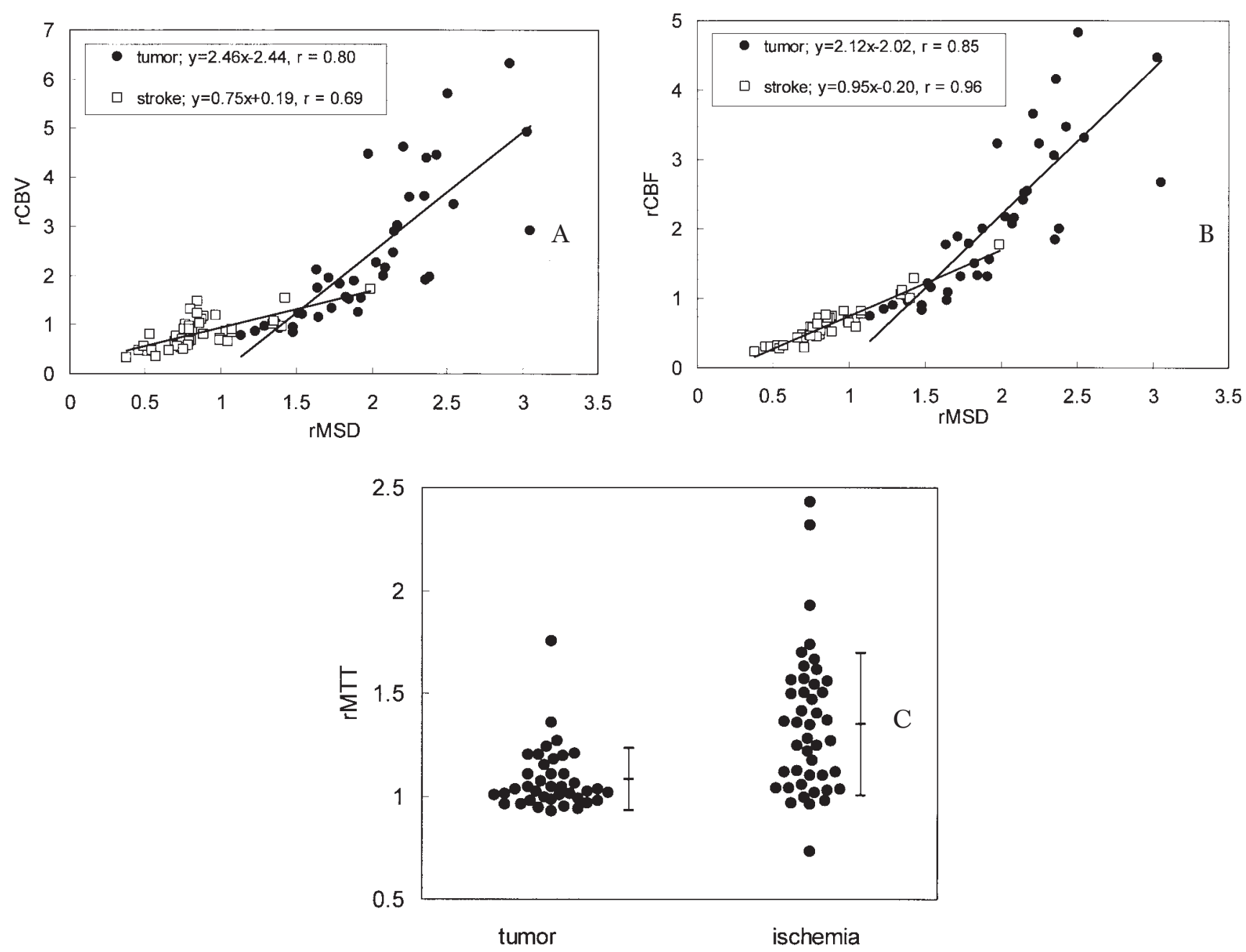

Fig 3. Relationships between relative maximum signal drop (rMSD) and 2 cerebral perfusion parameters for patients with cerebral gliomas (filled circles) or ischemic stroke (open squares). (A) Plot shows rMSD versus relative cerebral blood volume (rCBV). The data from 11 tumor patients showed a good association, with a Pearson's correlation coefficient of $0.80(\mathrm{n}=39)$, whereas those from 14 stroke patients showed only fair correlation $(r=0.69, \mathrm{n}=44)$. (B) Plot shows rMSD versus relative cerebral blood flow (rCBF). For the tumor group, the correlation was similar to that found in $\mathrm{A}(r=0.85)$. Data obtained from stroke patients, on the other hand, showed a strong association, reflected by a Pearson's correlation coefficient of 0.96. (C) Graph compares alterations in relative mean transit time (rMTT) on patients with cerebral gliomas and ischemic stroke. Stroke lesions showed significantly greater rMTT prolongation with wider variations compared to tumor lesions $(1.351 \pm 0.345 \mathrm{vs} 1.082 \pm 0.152, P<.0001$, Student's $t$ test). The use of rMSD to represent rCBV, therefore, may not be suitable for ischemic lesions. Bars indicate the range of data within $1 \mathrm{SD}$ above and below the mean values.

Results from both the patient studies and the computer simulations suggest that the rMSD values reflect $\mathrm{rCBF}$ better than rCBV. As stated in a previous section, the discrepancy between $\mathrm{rMSD}$ and $\mathrm{rCBV}$ is anticipated to arise from variations in contrast bolus dispersion that approximately parallel rMTT. To investigate this effect, the extent of contrast bolus dispersion was examined by retrieving the rMTT distribution in both patient groups. Figure 3C shows the result, which demonstrates that rMTT prolongation in ischemic stroke occurred to a greater extent $(1.351 \pm 0.345$, mean $\pm \mathrm{SD})$ than in cerebral gliomas $(1.082 \pm 0.152)$. The difference between the two groups was statistically significant $(P<.0001$, Student's $t$ test). In addition, ischemic lesions showed a much wider rMTT variation (larger SDs) than that in tumors. As a result, rMSD in ischemic tissues showed much weaker correlation with $\mathrm{rCBV}$ than in gliomas, consistent with the result in Figure 3A.

Figure 4A shows the rMTT map of a 52-year-old female patient with middle cerebral artery occlusion. It is noted that the infarct region showed significantly prolonged rMTT compared with normal parenchyma, presumably reflecting the existence of collateral circulation. ${ }^{17,18}$ The rMSD, rCBV, and $\mathrm{rCBF}$ maps of the same 

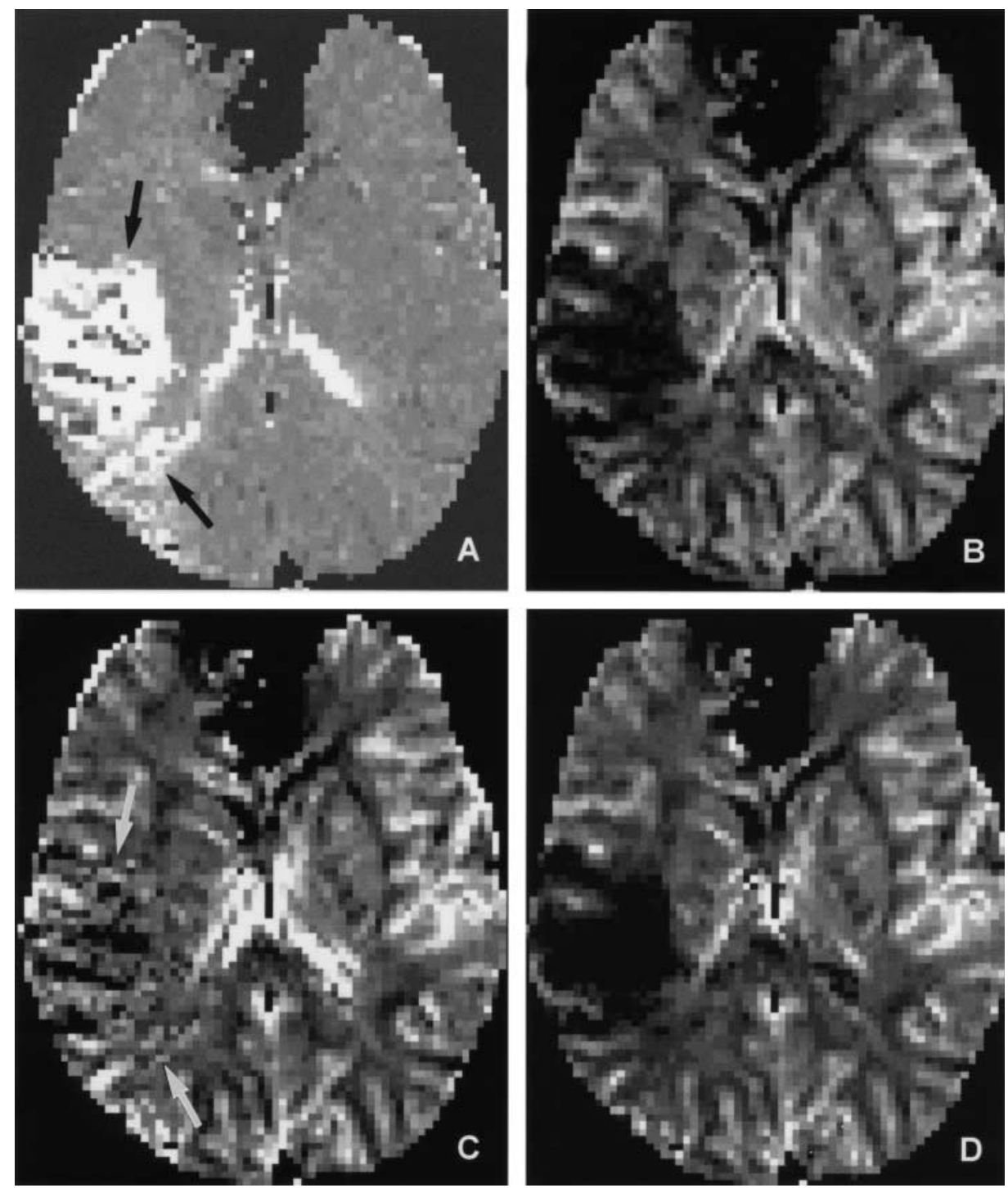

Fig 4. A 52-year-old woman with middle cerebral artery occlusion. (A) Transaxial image of relative mean transit time (rMTT) shows an area with abnormal hyperintensity (arrow), representing the existence of collateral flow in the ischemic lesion, causing a prolonged rMTT and hence a widened concentration-time curve. (B) Transaxial image of relative maximum signal drop (rMSD) obtained at the corresponding slice location, showing hypoperfusion in the area of prolonged rMTT. (C) Transaxial image of relative cerebral blood volume ( $\mathrm{rCBV}$ ) carefully adjusted to similar window settings shows inconsistency of $\mathrm{rCBV}$ with rMSD in the ischemic lesion (arrow). (D) Transaxial image of relative cerebral blood flow shows overall similarity to the rMSD map in B.

slice are displayed in Figures 4B, 4C, and 4D, respectively, with image intensity carefully adjusted to similar window settings. The inconsistency between $\mathrm{rMSD}$ and $\mathrm{rCBV}$ appeared to exist in the ischemic region with higher rMTT (arrows), consolidating the reasoning stated in the previous section. In contrast, Figures $5 \mathrm{~A}$ to $5 \mathrm{D}$ show the rMTT, rMSD, rCBV, and $\mathrm{rCBF}$ maps from a patient with oligodendroglioma. Since contrast bolus dispersion was relatively uniform, there existed little difference between rMSD and rCBV maps, except in diffusive spots in the tumor center showing prolonged rMTT (arrows). Also note that the rMSD map shown in Figure 5B exhibits a visually better contrast-to-noise ratio than the $\mathrm{rCBV}$ map in Figure 5C. ${ }^{8}$

\section{Discussion}

The results of this study indicate that although rMSD was reported to correlate with $\mathrm{rCBV}$ in patients with intracranial tumors, ${ }^{8,9}$ such a relationship does not necessarily hold for stroke patients. The result should be intuitive because $\mathrm{rCBV}$ obtained via numerical integration mirrors the total amount of contrast agents flowing through the tissue, whereas rMSD reflects information 

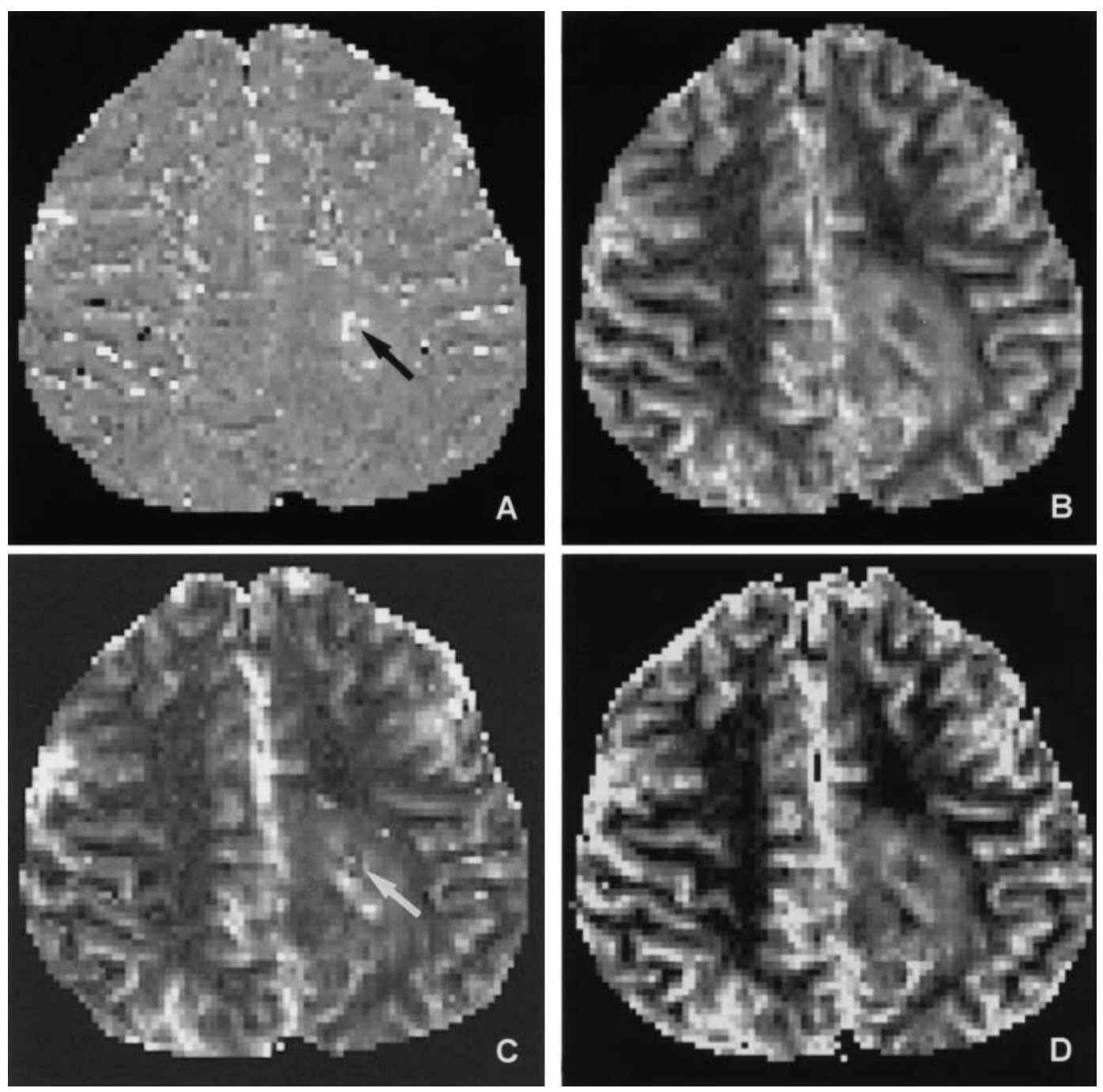

Fig 5. A 29-year-old man with pathologically proven oligodendroglioma imaged presurgically. (A) Transaxial image of relative mean transit time (rMTT) shows relatively uniform bolus dispersion throughout the tumor and normal brain parenchyma, indicating the absence of significant rMTT prolongation, except in diffusive spots in the tumor center (arrow). (B) Transaxial image of relative maximum signal drop (rMSD) obtained at the corresponding slice location. (C) Transaxial image of relative cerebral blood volume provides similar information to the rMSD map in B but with an inferior contrast-to-noise ratio. Diffusive spots in the tumor center showing large rMTT values are the only exceptions (arrow). (D) Transaxial image of relative cerebral blood flow again shows overall similarity to the rMSD map in B.

only on maximum tracer concentration. The claim that rMSD well predicts rCBV is therefore based on the important assumption that the width of the first-pass concentration-time curve must remain unaltered. Such phenomena predicted by our theoretical analysis were experimentally verified on patients (Fig 3A) and supported by simulation results (Fig 2A). Attention should thus be paid when applying the concept of rMSD in clinical practice.

Dispersion of the contrast bolus could originate from several sources. A slow injection speed may result in wide profiles of the first-pass transit, whose effect on the rMSDrCBV inconsistency can nevertheless be minimized, for example, by taking ratios of lesion rMSD to a contralateral homologous region. ${ }^{8}$ Focal alterations in the dispersion of contrast bolus due to hemodynamic changes, on the other hand, are difficult to remedy. For cerebral diseases related to hypoperfusion, such as ischemic stroke, ${ }^{19}$ vasospasm, ${ }^{20}$ edema,${ }^{21}$ or migrainous aura, ${ }^{22}$ the existence of collateral circulation is likely to lead to delayed contrast arrival. ${ }^{17}$ Concomitant prolongation in $\mathrm{rMTT}^{14}$ thus results in a broadening of the concentration-time curve. Moreover, the extent of bolus dispersion may be highly variable locally. The value of rMSD to represent rCBV in these diseases, consequently, is anticipated to be limited.

Regardless of the limitations addressed above, two previous reports ${ }^{8,9}$ suggested that at least for patients with 
cerebral tumors, rMSD turns out to be a good alternative to obtain equivalent $\mathrm{rCBV}$ information. In cerebral gliomas, a focal increase in tissue vascularity due to angiogenesis leads to elevated $\mathrm{rCBV}^{5-7}$ Since the upstream arterial pathway remains relatively unaltered, rMTT in tumors tends to be similar to normal brain parenchyma. As a result, rCBV elevation in cerebral tumors is largely reflected by an increase in rMSD, supporting the validity of the rMSD method. In other words, the $\mathrm{rMSD}$-rCBV relationship reported previously ${ }^{8,9}$ is disease specific and strongly depends on the hemodynamics involved in the pathophysiological processes.

Both the patient data in Figure $3 \mathrm{~B}$ and the computer simulation results in Figure 2B confirm our theoretical inference that $\mathrm{rMSD}$ actually better represents $\mathrm{rCBF}$, regardless of the possible existence of rMTT prolongation. Similar results of peak tracer concentration to represent region blood flow have been demonstrated in cardiac nuclear medicine studies in which during the first-pass transit, there was negligible tracer clearance into the veins. ${ }^{23}$ Even if the condition of slow tracer clearance may not be met in general for dynamic susceptibility contrast magnetic resonance perfusion studies, our patient data suggest that the use of rMSD to represent $\mathrm{rCBF}$ should be valid.

For tumor lesions, data scattering was found in both the rMSD-rCBV and rMSD-rCBF relationships (Figs 3A and 3B). In other words, the representative value of rMSD for $\mathrm{rCBF}$ is no better than for $\mathrm{rCBV}$ in gliomas. The scattering found in the tumor rMSD-rCBF relationship but absent in the simulation results is anticipated to arise from an alteration in the tissue vasculature, leading to deviations of the concentration-time curve from a gamma variate shape. ${ }^{24}$ Under such circumstances, the tumor vasculature often renders overlap of the first-pass transit and recirculation phases of the concentration-time curve. Difficulty in the definition of $\mathrm{rCBF}$ can be encountered in these cases depending on the degree of vessel tortuosity, hence resulting in $\mathrm{MMSD}-\mathrm{rCBF}$ data scattering, as in Figure 3B.

In conclusion, $\mathrm{rMSD}$ correlates with $\mathrm{rCBV}$ in patients with cerebral tumors. In examples in which substantial rMTT prolongations were present, $\mathrm{rMSD}$ reflects $\mathrm{rCBF}$ information rather than $\mathrm{rCBV}$. Consequently, with superior noise immunity, rMSD is an effective and convenient method for a quick visualization of cerebral hemodynamics. The use of rMSD, however, demands caution when interpreting different cerebral hemodynamic pathophysiology.

\section{References}

1. Sorensen AG, Tievsky AL, Ostergaard L, Weisskoff RM, Rosen BR. Contrast agents in functional MR imaging. $J$ Magn Reson Imaging 1997;7:47-55.

2. Petrella JR, Provenzale JM. MR perfusion imaging of the brain: techniques and applications. Am J Roentgenol 2000;175:207-219.

3. Belliveau JW, Rosen BR, Kantor HL, et al. Functional cerebral imaging by susceptibility-contrast NMR. Magn Reson Med 1990;14:538-546.

4. Rosen BR, Belliveau JW, Buchbinder BR, et al. Contrast agents and cerebral hemodynamics. Magn Reson Med 1991;19:285-292.

5. Aronen HJ, Gazit IE, Louis DN, et al. Cerebral blood volume maps of gliomas: comparison with tumor grade and histologic findings. Radiology 1994;191:41-51.

6. Sugahara T, Korogi Y, Kochi M, et al. Correlation of MR imaging-determined cerebral blood volume maps with histologic and angiographic determination of vascularity of gliomas. Am J Roentgenol 1998;171:1479-1486.

7. Knopp EA, Cha S, Johnson G, et al. Glial neoplasms: dynamic contrast-enhanced $\mathrm{T} 2 *$-weighted MR imaging. Radiology 1999;211:791-798.

8. Cha S, Lu S, Johnson G, Knopp EA. Dynamic susceptibility contrast MR imaging: correlation of signal intensity changes with cerebral blood volume measurements. J Magn Reson Imaging 2000;11:114-119.

9. Berchtenbreiter C, Bruening R, Wu RH, Penzkofer H, Weber J, Reiser M. Comparison of the diagnostic information in relative cerebral blood volume, maximum concentration, and subtraction signal intensity maps based on magnetic resonance imaging of gliomas. Invest Radiol 1999;34:75-81.

10. Thompson HK, Starmer CF, Whalen RE, Mcintosh H. Indicator transit time consider as a gamma variable. Circ Res 1964;14:502-515.

11. Berninger WH, Axel L, Norman D, Napel S, Redington RW. Functional imaging of the brain using computed tomography. Radiology 1981;138:711-716.

12. Schreiber WG, Guckel F, Stritzke P, Schmiedek P, Schwartz A, Brix G. Cerebral blood flow and cerebrovascular reserve capacity: estimation by dynamic magnetic resonance imaging. J Cereb Blood Flow Metab 1998;18:1143-1156.

13. Weisskoff RM, Chesler D, Boxerman JL, Rosen BR. Pitfalls in MR measurement of tissue blood flow with intravascular tracers: which mean transit time? Magn Reson Med 1993;29:553-559.

14. Ostergaard L, Weisskoff RM, Chesler DA, Gyldensted C, Rosen BR. High resolution measurement of cerebral blood flow using intravascular tracer bolus passages. Part I: Mathematical approach and statistical analysis. Magn Reson Med 1996;36:715-725.

15. Axel L. Cerebral blood flow determination by rapidsequence computed tomography. Radiology 1980;137:679686.

16. Henkelman RM. Measurement of signal intensities in the presence of noise in MR images. Med Phys 1985;12:232-233.

17. Kluytmans M, van der Grond J, van Everdingen KJ, Klijn CJ, Kappelle LJ, Viergever MA. Cerebral hemodynamics in 
relation to patterns of collateral flow. Stroke 1999;30:14321439.

18. Lythgoe DJ, Ostergaard L, William SC, et al. Quantitative perfusion imaging in carotid artery stenosis using dynamic susceptibility contrast-enhanced magnetic resonance imaging. Magn Reson Imaging 2000;18:1-11.

19. Sorensen AG, Buonanno FS, Gonzalez RG, et al. Hyperacute stroke: evaluation with combined multisection diffusion-weighted and hemodynamically weighted echoplanar MR imaging. Radiology 1996;199:391-401.

20. Rordorf G, Koroshetz WJ, Copen WA, et al. Diffusion- and perfusion-weighted imaging in vasospasm after subarachnoid hemorrhage. Stroke 1999;30:599-605.

21. Stoll M, Hagen T, Bartylla K, Weber M, Jost V, Treib J. Changes of cerebral perfusion after osmotherapy in acute cerebral edema assessed with perfusion weighted MRI. Neurol Res 1998;20:474-478.

22. Cutrer FM, Sorensen AG, Weisskoff RM, et al. Perfusionweighted imaging defects during spontaneous migrainous aura. Ann Neurol 1998;43:25-31.

23. Mullani NA, Gould KL. First-pass measurements of regional blood flow with external detectors. J Nucl Med 1983;24:577-581.

24. Kassner A, Annesley DJ, Zho XP, et al. Abnormalities of the contrast re-circulation phase in cerebral tumors demonstrated using dynamic susceptibility-enhanced imaging: a possible marker of vascular tortuosity. J Magn Reson Imaging 2000;11:103-113. 